\title{
An Update on Preoperative Radiotherapy for Locally Advanced Rectal Cancer
}

\author{
Seung-Gu Yeo, Dae Yong Kim ${ }^{1}$ \\ Department of Radiation Oncology, Soonchunhyang University College of Medicine, Cheonan; ${ }^{1}$ Center for Colorectal Cancer, Research \\ Institute and Hospital, National Cancer Center, Goyang, Korea
}

Even in patients undergoing an optimal surgical technique (e.g., total mesorectal excision), radiotherapy provides a significant benefit in the local control of rectal cancer. Compared with postoperative treatment, chemoradiotherapy given preoperatively has been shown to decrease local recurrence rates and toxicity. Additionally, preoperative chemoradiotherapy permits the early identification of tumor responses to this cytotoxic treatment by surgical pathology. Pathological parameters reflecting the tumor response to chemoradiotherapy have been shown to be surrogate markers for long-term clinical outcomes. Post-chemoradiotherapy downstaging from cStage II-III to ypStage 0-I indicates a favorable prognosis, with no difference between ypStage 0 and ypStage I. Research is ongoing to develop useful tools (clinical, molecular, and radiological) for clinical determination of the pathologic chemoradiotherapeutic response before surgery, and possibly even before preoperative treatment. In the future, risk-adapted strategies, including intensification of preoperative therapy, conservative surgery, or the selective administration of postoperative chemotherapy, will be realized for locally-advanced rectal cancer patients based on their response to preoperative chemoradiotherapy.

\section{Keywords: Rectal neoplasms; Radiotherapy; Chemoradiotherapy; Neoadjuvant}

\section{INTRODUCTION}

In contrast to colon cancer, several features make the successful management of rectal cancer clinically challenging. The absence of a serosal barrier permits early tumor extension into the perirectal tissues. The rectum also lies close to vital, and sometimes unresectable, pelvic structures (e.g., the bladder, ureters, iliac vessels, and the sacrum). The compact nature of the mesorectum within the confines of the pelvis complicates the adequate removal of all mesenteric nodes at risk for metastatic disease [1]. Due to these specific characteristics of rectal cancer, surgery alone has been associated historically with local recurrence in up to one in four pa-

Received: June 27, 2012 - Accepted: August 9, 2012

Correspondence to: Dae Yong Kim, M.D.

Center for Colorectal Cancer, National Cancer Center, 323 Ilsan-ro,

Ilsandong-gu, Goyang 410-769, Korea

Tel: +82-31-920-1721, Fax: +82-31-920-0149

E-mail: radiopiakim@hanmail.net

(c) 2012 The Korean Society of Coloproctology

This is an open-access article distributed under the terms of the Creative Commons Attribution NonCommercial License (http://creativecommons.org/licenses/by-nc/3.0) which permits unrestricted noncommercial use, distribution, and reproduction in any medium, provided the original work is properly cited. tients. Locally recurrent disease is usually incurable and causes profound morbidity, suffering, and death. Subsequent adjuvant randomized trials evaluated different combinations of radiation therapy (RT) and 5-fluorouracil-based chemotherapy. These studies showed that postoperative chemoradiotherapy (CRT) improved survival and local control relative to surgery alone, leading to the standard implementation of these therapies for locally-advanced rectal cancer (LARC) by the 1990s [2, 3].

Because of the potential benefits associated with preoperative therapy, interest has evolved in adopting this approach in rectal cancer patients. A randomized trial from Europe proved that by simply altering the sequence of CRT, improved rates of local control, treatment compliance, and acute and late toxicity could be achieved, leading to a new paradigm of care [4]. Parallel to this paradigm shift in treatment sequence, diverse CRT responses among patients can be identified earlier in surgical specimens whereas these responses could only have been identified after long-term follow-up when CRT was provided postoperatively. Accordingly, several questions have newly emerged regarding these diverse pathologic CRT responses: Can a good pathologic CRT response be an early indicator of good prognosis? How can we define the criteria for a good pathologic CRT response? Can we predict a good 
pathologic CRT response before surgery or before preoperative CRT? Following preoperative CRT, is less invasive or less radical surgery feasible for patients with a good CRT response? Herein, we review these questions that are confronted in the preoperative CRT approach for patients with LARC.

\section{PREOPERATIVE CRT}

Preoperative RT has been shown to significantly decrease the local recurrence rate and improve survival, provided that a biologically equivalent dose of at least $30 \mathrm{~Gy}$ is administered [5]. The potential advantages of preoperative over postoperative RT include enhanced effectiveness in well-oxygenated tissue, a damaging effect on cells that may be spread locally or distantly at the time of resection, downstaging of advanced tumors, and better treatment compliance [6]. This theoretical superiority of the preoperative approach over postoperative adjuvant therapy has been confirmed in the German rectal cancer trial (CAO/ARO/AIO 94 trial) [4]. This trial randomly assigned more than 800 patients with cT3-4 or $\mathrm{cN}+$ rectal cancer to two different CRT sequence strategies. Compared with postoperative therapy, patients who received preoperative therapy had a significantly lower incidences of local recurrence ( $15 \%$ vs. $6 \%, \mathrm{P}=0.006$ ), acute toxicity ( $40 \%$ vs. $27 \%, \mathrm{P}=0.001$ ) and chronic toxicity ( $24 \%$ vs. $14 \%, \mathrm{P}=0.012)$, a higher incidence of sphincter preservation ( $20 \%$ vs. $39 \%, \mathrm{P}=0.005)$, and no difference in 5 -year overall survival (OS; 76\% vs. 74\%). The CAO/ARO/ AIO 94 trial remains the definitive trial in CRT for rectal cancer.

Although the results must be interpreted cautiously due to the trial's limitations, other studies comparing the two different CRT sequences have reported improved disease-free survival (DFS) with no difference in local control [7, 8]. The National Surgical Adjuvant Breast and Bowel Project R-03 study [7] had limitations regarding premature closure of the trial due to insufficient accrual and used different chemotherapy regimens and surgical techniques compared with the CAO/ARO/AIO 94 trial. However, patients with LARC who received preoperative therapy showed a significant improvement in 5-year DFS compared with those who received postoperative therapy ( $65 \%$ vs. $53 \%, \mathrm{P}=0.011)$. Local recurrence rates did not differ (11\%) between the preoperative and the postoperative approaches. Similar outcomes were reported by Yeo et al. [8], although their study was also limited by its retrospective nature. They analyzed 487 patients with cT3 mid or distal rectal cancer who had received preoperative or postoperative CRT. The 5-year local-recurrence-free survival rate was not different, 95.3\% and $92.1 \%$ in the preoperative and the postoperative CRT groups, respectively $(\mathrm{P}=0.402)$; however, the 5 -year DFS rate of $78.8 \%$ in the preoperative CRT group was significantly better than that of $63.0 \%$ in the postoperative CRT group $(\mathrm{P}=0.002)$.

During the same period, similar to improvements in adjuvant treatments, improvements in surgical techniques have dramatically lowered the incidence of local recurrence in rectal cancer. Careful pathological studies have clearly demonstrated that the major cause of local recurrence is the persistence of tumor foci within the mesorectum [9]. Intact removal of the entire mesorectum (total mesorectal excision, TME) in cancers of the mid or lower third of the rectum was pioneered by Heald et al. [10] and has resulted in local recurrence rates lower than 5-10\%.

However, the effect of preoperative RT on the local recurrence rate was consistent even when the optimal surgical technique (TME) was implemented. This was demonstrated by the results of a Dutch rectal cancer trial that randomized 1,861 rectal cancer patients to undergo either RT followed by TME or TME alone in the setting of a national surgical training program [11]. Compared with TME alone, a short-course ( 5 Gy $\times 5$ fractions) of RT followed by TME within a week resulted in a significantly lower local recurrence rate, although no improvement in OS was noted. The 10-year cumulative incidence of local recurrence was $5 \%$ in the group assigned to RT and surgery and $11 \%$ in the surgery-alone group $(\mathrm{P}<0.0001)$; i.e., preoperative RT reduced the 10 -year local recurrence by more than $50 \%$ relative to surgery alone [11]. A similar benefit in local control with preoperative RT combined with TME was shown by a Medical Research Council trial, which evaluated routine preoperative short-course RT versus selected (restricted to patients with involvement of the circumferential resection margin) postoperative CRT [12].

The trials from the European Organization for Research and Treatment of Cancer and Fédération Francophone de la Cancérologie Digestive have supported the results of previous trials, finding that improved pathologic complete response rates and local control can be achieved with the addition of chemotherapy to (long-course) RT, although the survival impact of concurrent chemotherapy in a preoperative approach remains uncertain $[13,14]$.

\section{PROGNOSTIC SIGNIFICANCE OF A PATHOLOGIC CRT RESPONSE}

In the postoperative CRT era, tumor response to CRT could be determined after long-term follow-up; however, if preoperative CRT is employed, tumor response can be assessed much earlier in the surgical specimen. For most patients, preoperative CRT results in clinically meaningful tumor regression, but the degree of response varies among patients: some show almost no response whereas others exhibit a pathologic complete response (ypCR). The ypCR with absence of viable tumor cells in the surgical specimen occurs in approximately $10-30 \%$ of LARC patients. Although some studies have reported no improvement in outcomes for patients with ypCR [15], many have suggested that ypCR is associated with a favorable outcome regarding local control, distant recurrence, DFS, and OS [16-18]. One explanation for the good prognosis after ypCR is that ypCR is indicative of a prognostically favorable biological tumor profile, with lower propensity for local recurrence and distant metastases and better survival than for patients showing a lesser response.

The Korean Radiation Oncology Group (KROG 09-01) collected 
clinical data for 333 LARC patients with ypT0Nany following preoperative CRT and curative radical resections between 1993 and 2007 [17]. After a median follow-up of 43 months, the 5-year DFS was $84.6 \%$ and OS was $92.8 \%$. Excluding 29 patients (8.7\%) with ypT0N+ disease, the 5-year DFS and OS were $88.5 \%$ and $94.8 \%$, respectively, in 304 ypT0N0 patients. Similarly, an Italian group reported the prognostic value of ypCR in a study of $566 \mathrm{ypT0N0}$ patients from 36 centers [16]. After a median follow-up of 46 months, pelvic relapse and distant metastasis occurred in 1.6\% and $8.9 \%$, respectively. The 5-year DFS, OS, and cancer-specific survival rates were $85 \%, 90 \%$, and $94 \%$, respectively. These studies showed that LARC patients achieving ypT0N0 status after preoperative CRT had favorable long-term outcomes.

Favorable outcomes for ypCR patients were evident when compared with a control group with residual disease. Maas et al. [18] performed a pooled analysis of individual patient data from several study centers, and 484 (15.6\%) of 3,105 patients showed ypCR. The median follow-up for all patients was 48 months, and the 5year crude DFS was $83.3 \%$ for patients with ypCR and $65.6 \%$ for those without ypCR $(\mathrm{P}<0.0001)$. The adjusted hazard ratio for ypCR for failure was 0.54 (95\% confidence interval, 0.40-0.73), indicating that patients with ypCR had a significantly increased probability of DFS. The effect of ypCR on long-term outcome was not affected or modified by clinical $\mathrm{T}$ or $\mathrm{N}$ category, administration of postoperative chemotherapy, distance from the anal verge, or type of surgery.

\section{CRITERIA FOR GOOD RESPONSE}

The data indicate that pathologically assessed tumor response to preoperative therapy can be a short-term treatment response indicator and a surrogate marker of long-term outcome. Accordingly, novel preoperative treatments may be compared with benchmarks of response-stratified outcomes. Knowledge of such benchmarks may also be useful for clinicians to develop subsequent treatments allowing risk-adapted strategies. However, some discussion is necessary regarding the criteria of benchmarks, namely, good pathologic response: Does ypCR include complete response in the primary tumor only (ypTONany) or should it be restricted to ypT0N0? Is there a prognostic difference between ypCR and minimal residual disease?

The ypN status in LARC patients receiving preoperative CRT demonstrated major prognostic importance. Among the four factors (ypT, ypN, ypStage, and Dworak's tumor regression grade [TRG]), which were found to be significant predictors of DFS in a multivariate analysis, ypN status was the most discriminatory factor predicting DFS, followed by ypStage, ypT, and finally TRG [19]. Another study similarly reported that the ypN classification was the most significant factor independently predicting the OS of LARC patients [20]. The equally important prognostic significance of ypN status was also found in selected patients with ypT0 status. In the KROG 09-01 study [17], ypN status was ypT0N0 in 304 patients
(91.3\%), ypT0N1 in 22 patients (6.6\%), and ypT0N2 in 7 patients (2.1\%). A patient's ypN status was revealed to be the most relevant independent prognostic factor for both DFS and OS in $333 \mathrm{ypT0}$ patients: 5-year DFS rates were $88.5 \%, 51.9 \%$, and $21.4 \%$, and the 5 -year OS rates were $94.8 \%, 76.2 \%$, and $64.3 \%$ in ypT0N0, ypT0N1, and ypT0N2 patients, respectively ( $<0.001$ for both). ypT0N+ patients constitute a very small proportion of the ypT0 group; thus, they may not change to any great degree the favorable outcomes of ypT0Nany patients. However, patients with ypT0N+ disease were in a separate group with a distinctly unfavorable prognosis, so the criteria of good pathologic response need to be restricted to ypT0N0 status [21].

When the most important prognostic factor in LARC receiving preoperative CRT, the ypN status, is the same as the ypN0 status, minimal residual disease in the primary tumor (ypT1-2N0) may not confer a significantly different prognosis compared with ypT0N0 status. Moon et al. [22] showed that patients with ypT0N0, ypT1N0, and ypT2N0 cancers demonstrated no significant difference in 5-year OS (ypT0N0, 96.5\%; ypT1N0, 95.7\%; ypT2N0, 92.4\%; P $=0.497$ ) or 5-year DFS (ypT0N0, 90.2\%; ypT1N0, 100\%; ypT2N0, 89.3\%; P = 0.205). In terms of ypStage, the 5-year OS (ypStage 0, 96.5\%; ypStage I, 92.9\%; $\mathrm{P}=0.346$ ) and 5-year DFS (ypStage 0, 90.2\%; ypStage I, 90.7\%; $\mathrm{P}=0.879$ ) were no better in patients with ypStage 0 tumors than in those with ypStage I tumors. The authors insisted that a suitable endpoint representing a good pathologic CRT response would be a grouping that combines ypStage 0 and ypStage I (ypT0-2N0M0).

In a stage-to-stage (ypStage vs. pStage) comparative analysis that demonstrated better DFS after preoperative CRT compared with postoperative CRT for $\mathrm{CT} 3$ rectal cancer patients [8], preoperative CRT yielded a downstaging to ypStage 0-I in 155 patients (42.6\%), and this downstaged group showed a significantly improved DFS compared not only with ypStage II but also with pStage II patients with postoperative CRT. Post-CRT ypStage 0-I patients revealed outcomes that were nearly identical to the historical results for pStage I rectal cancer without postoperative treatment. The ypStages II and III, in which patients with and without CRT responses in the primary tumor and/or mesorectal lymph nodes were combined, demonstrated DFSs that were similar to those for pStages II and III, respectively. Thus, the existence of ypStage 0-I patients was suggested to be the cause of the improved DFS observed in the preoperative CRT group.

\section{PREDICTION OF GOOD RESPONSE}

In contrast to postoperative treatments that can be modified based on pathologic CRT response information, markers predicting CRT response are necessary to implement risk-adapted strategies in preoperative treatment and surgery. More aggressive preoperative regimens may be considered in patients who are less likely to respond to standard preoperative therapy. Conversely, sphincter-sparing surgical procedures or tumor-localized resections may be an 


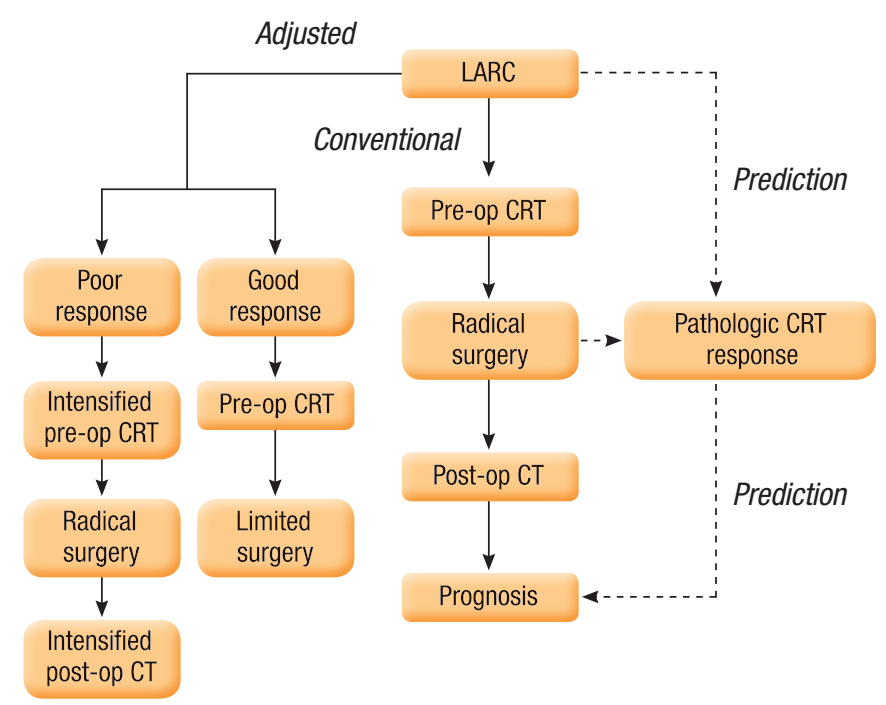

Fig. 1. Schematic diagram of multimodality treatments for patients with locally advanced rectal cancer (LARC). Conventional treatments for LARC consisted of preoperative chemoradiotherapy (CRT), radical surgery, and postoperative chemotherapy (CT). When the pathologic CRT response reliably predicting long-term outcomes can be accurately determined before surgery or before CRT, then selected patients with good or poor CRT response may be recommended for less or more intensified treatments, respectively, rather than conventional treatments. Pre-op, preoperative; Post-op, postoperative.

alternative to standard radical surgery in selected patients who show excellent tumor regression after preoperative CRT (Fig. 1). This patient-tailored approach demands the availability of reliable information concerning biological tumor behavior, the expected response to treatment, or post-CRT disease status before surgery. Research is actively ongoing to identify useful tools for predicting pathologic CRT response.

\section{Clinical factors}

Carcinoembryonic antigen (CEA) has been shown to inhibit cell death by causing a loss of anchorage to the extracellular matrix [23]. Tumor cells containing a high density of CEA may resist radiation-induced changes that occur using either a pro-apoptotic or pro-survival molecular pathway. Yoon et al. [24] investigated pre-CRT clinical parameters that could predict CRT response in LARC. Multivariate analysis revealed the following: predictors of T-level downstaging (ypT0-2) included pretreatment hemoglobin level, cN0 classification, and serum CEA level; predictors of tumor regression (TRG 3-4) included $\mathrm{cN} 0$ classification and CEA level; and the predictor of complete regression was the CEA level. The authors suggested that the pre-CRT CEA level $(\leq 5 \mathrm{ng} / \mathrm{mL}$ vs. $>5 \mathrm{ng} / \mathrm{mL}$ ) was the most important clinical predictor of pathologic tumor response. Furthermore, Park et al. [25] analyzed changes in the ratios of tumor response with increasing CEA levels in LARC. The rates of good response (TRG 3-4) decreased sig- nificantly as the pre-CRT CEA levels became more elevated (CEA, ng/mL: $\leq 3,36.4 \%$; 3-6, 23.6\%; 6-9, 15.6\%; >9, 7.8\%; P < 0.001), revealing a linear inverse correlation between pre-CRT CEA levels and tumor response.

Hypoxia is associated with resistance to RT. Tumor oxygenation is affected by several factors, such as adequacy of blood supply, microcirculation, and the oxygen-carrying capacity of the blood, which is expressed as the hemoglobin level. Lee et al. [26] reported that tumor response rates in LARC were significantly different below and above a hemoglobin level of $9.0 \mathrm{~g} / \mathrm{dL}$. Favorable tumor response (TRG 3-4) was shown in $29 \%$ of patients with hemoglobin levels $\geq 9.0 \mathrm{~g} / \mathrm{dL}$, but in no patient with a hemoglobin level $<9.0 \mathrm{~g} / \mathrm{dL}$. Additionally, no difference was observed in tumor response between the non-transfusion and the transfusion groups of patients with hemoglobin levels $\geq 9.0 \mathrm{~g} / \mathrm{dL}$. The authors suggested that pre-CRT anemia is an indicator of poor response to preoperative CRT and that transfusion may improve its therapeutic efficacy.

\section{Molecular markers}

Studies on molecular biomarkers for predicting the response to preoperative CRT in rectal cancer have focused on tumor suppressor genes (p53, p21), apoptotic factors (Bcl-2, Bax), epidermal growth factor receptor, cyclooxygenase-2 (COX-2), and vascular endothelial growth factor, or on microarray gene expression analysis displaying complete genomic patterns [27]. Bax is a pro-apoptotic protein, and its expression induces mitochondrial permeability, which leads to release of cytochrome $\mathrm{c}$ and initiation of a signaling cascade promoting DNA degradation and cell death. Bax expression in rectal tumors was found to be significantly higher in the post-CRT complete response group than in the partial response group (54\% vs. $29 \%, \mathrm{P}=0.017$ ) [28]. Overexpression of COX-2 in pretreatment rectal tumors was shown to be predictive of poor tumor regression after preoperative CRT, and a clinical trial of COX-2 inhibitors for patients with COX-2 overexpression was suggested to improve the response rate of preoperative CRT [29].

A single molecular marker approach has limitations in therapeutic response prediction, and no single marker has yet been consistently identified as clinically applicable in rectal cancer. By contrast, microarray gene expression analysis, which enables the display of complete genome patterns at one time, may be more promising. Kim et al. [30] obtained tumor tissues from 46 patients with rectal cancer (31 for training and 15 for validation testing). The 31 training samples comprised 20 partial CRT response and 11 complete CRT response cases. A primary set of 261 genes was identified as differentiating between partial and complete response. A gene set comprising the top-ranked 95 genes, displaying differential expression between partial and complete response, was applied to predict response to CRT. Complete response and partial response were accurately predicted in $84 \%(26 / 31)$ of the training samples and $87 \%(13 / 15)$ of the validation samples. 


\section{Imaging}

The variability in tumor-size changes after preoperative CRT may represent an aspect of biologic tumor heterogeneity that can yield important information for prognostic prediction in LARC patients. However, irregular tumor shape and non-uniform treatment-related shrinkage make it difficult to identify all subtle tumor-size changes induced by CRT using traditional methods, which measure two-dimensional (World Health Organization) or only onedimensional (Response Evaluation Criteria in Solid Tumors) maximal tumor diameters [31]. This challenge can be overcome by quantifying detailed changes in tumor volume using three-dimensional region-of-interest volumetry. Yeo et al. [32] measured tumor volume using three-dimensional region-of-interest volumetry based on magnetic resonance imaging (MRI) before and after preoperative CRT, but before surgery. They analyzed the correlation between the tumor volume reduction rate (TVRR) and the pathologic tumor response in 405 LARC patients. The mean TVRR was $65.0 \% \pm 22.3 \%$ for all patients. TVRRs according to ypT classification (ypT0-T2 vs. ypT3-T4), ypN classification (ypN0 vs. ypN+), downstaging (ypStage 0-I vs. ypStage II-III), good regression (TRG 3-4 vs. TRG 1-2), and complete regression (TRG 4 vs. TRG 1-3) were all significantly different $(\mathrm{P}<0.05)$. When the TVRR was categorized into three groups $(<60 \%, 60-80 \%$, and $>80 \%$ ), the rates of ypT0-T2, ypN0, downstaging, and good regression were all significantly greater for patients with TVRRs $\geq 60 \%$, as was the complete regression rate for patients with TVRRs $>80 \%$ $(\mathrm{P}<0.05)$. In addition to its predictive value, this TVRR information also showed statistical significance as an independent prognostic factor for LARC patients [33]. Kang et al. [34] analyzed 84 LARC patients after similarly measuring the TVRR and reported that TVRR $>75 \%$ was significantly associated with increased ypCR rate, but that TVRR did not show a significant association with ypT or ypN down-classification and TRG. However, they used a different definition of ypT down-classification (ypT lower than cT) and a different TRG grading system (Mandard's).

Conventional imaging modalities have inherent limitations in assessing the response to preoperative CRT for rectal cancer due to post-radiation edema, inflammation, and fibrosis [35]. Instead, functional imaging techniques depict the metabolic and the microstructural characteristics of the tumor and can provide a deeper insight into the tumor's biological property. In rectal cancer, two imaging techniques show great promise for response prediction during and after preoperative treatment: namely, ${ }^{18}$ fluoro-deoxy glucose positron emission tomography-computed tomography $\left({ }^{18} \mathrm{~F}-\mathrm{FDG}\right.$ PET-CT) and diffusion-weighted MRI (DW-MRI). A significant reduction was noted in the standardized uptake value (SUV) on post-radiation PET of responders compared with nonresponders [36]. However, ${ }^{18}$ FDG-PET has some limitations, such as spatial resolution and difficulty in differentiating between tumor and inflammation. Conversely, DW-MRI can differentiate persistent tumors from therapy-related inflammation or necrosis with a high specificity. Patients with a good response to treatment generally show a lower apparent diffusion coefficient (ADC) prior to treatment, with a higher change in ADC during and after CRT [37]. The hypothesis is that a low initial ADC value represents a more restrictive environment with less interstitial edema and necrosis and a higher cellularity. Necrosis is generally associated with an acidic environment and a low oxygen concentration that influences the response to CRT. Lambrecht et al. [38] explored combining the above two imaging modalities for determining the probability of ypCR after preoperative CRT in LARC. The authors postulated the existence of a predictive algorithm for ypCR with a sensitivity of $100 \%$ and a specificity of $94 \%$ by combining the optimal $\triangle \mathrm{SUV}$ (max) thresholds during and after CRT or by combining the thresholds for the initial ADC value and the $\triangle \mathrm{SUV}(\max )$ during CRT. However, before these complex imaging techniques and consequent analyses can be implemented in daily practice, considerable effort towards standardization of protocols and analysis is necessary to ensure reproducibility of the results.

\section{LOCAL EXCISION IN GOOD RESPONDERS}

The mainstay of surgical therapy for LARC remains the low anterior resection or the abdominoperineal resection. Unfortunately, significant morbidity risks are associated with a radical resection, including frequent defecation, voiding problems, and sexual dysfunction. Furthermore, the lifestyle and the body image of patients who undergo an abdominoperineal resection are profoundly altered $[1,39]$. Patients who either refuse abdominoperineal resection to preserve the anal sphincter or have pre-existing medical co-morbidities have led some physicians to advocate for local excision in selected rectal cancer cases. However, local excision does not surgically address the lymphatic tissue at risk within the mesorectum. Thus, tumors at high risk for lymphatic spread or clinically obvious nodal involvement would not be readily amenable to this approach. For this reason, indications for local excision have been highly restricted to tumors without factors associated with mesorectal lymph node metastasis, such as cT2-3, high grade, lymphovascular/perineural invasion, $>3-4 \mathrm{~cm}$ in size, or $>40 \%$ of the rectal wall circumference [1].

Significant tumor regression (downsizing and downstaging) following preoperative CRT may lead to the use of local excision as an alternative treatment option even in patients with the above risk factors. The key rationale for this approach is the correlation between radiosensitivity and the inherited low aggressiveness of rectal cancer, and the correlation between the radiosensitivity of the primary tumor and that of the mesorectal nodal disease [40]. Read et al. [41] analyzed 644 patients with rectal cancer receiving preoperative RT/CRT and a proctectomy. The ypN+ rate was $2 \%$ in ypT0, $4 \%$ in ypT1, 23\% in ypT2, 47\% in ypT3, and $48 \%$ in ypT4 patients. The authors suggested that it was reasonable to select ypT0-1 for local excision and to reserve a proctectomy for patients who demonstrated residual ypT2-4 disease. Kim et al. [42] reported that $\mathrm{ypT}$ classification was the most reliable predictor of 
ypN status in a multivariate analysis including 282 LARC patients. The ypN+ rate was $3.4 \%$ in ypT0-1, $16.9 \%$ in ypT2, and $49.3 \%$ in ypT3 patients.

A prospective randomized study was undertaken to compare the oncologic results for local excision via transanal endoscopic microsurgery and those for laparoscopic TME in the treatment of cT2N0 rectal cancer following preoperative CRT [43]. Seventy patients were enrolled, and after a median follow-up of 84 months, both treatment groups demonstrated similar results in terms of local recurrences, distant metastases, and probability of survival. Yeo et al. [44] reported the outcomes of 11 patients with cT3 distal rectal cancer who received a local excision following preoperative CRT between 2003 and 2008. Local excision was recommended for patients who met all of the following criteria: 1) refusal of radical surgery or no acceptance of anal ablation, 2) initial cT3 classification, 3) clinically complete remission or minimal residual, and 4) full recognition of risks for local excision. The ypT0 was observed in eight patients, ypT1 in two patients, and ypT2 in one patient. After a median follow-up of 59 months, the 5-year local-recurrencefree survival, DFS, and OS rates were $90.9 \%, 81.8 \%$, and $88.9 \%$, respectively. No grade 3 or worse gastrointestinal toxicity was detected. Immediate radical surgery was strongly recommended for a patient with ypT2, but he refused and died of distant metastasis. The authors suggest that full-thickness local excision following CRT may be an acceptable option for cT3 distal rectal cancer that responds well to CRT. The M. D. Anderson Cancer Center performed a retrospective review of patients who underwent preoperative CRT followed by surgery for cT3 rectal cancer [45]. Fortyseven patients underwent local excision, and 473 patients underwent TME. Twenty-three (49\%) local excision patients had a complete response to CRT, 17 (36\%) had microscopic residual disease, and 7 (15\%) had gross residual disease, compared with 108 (23\%), 89 (19\%), and 276 (58\%) TME patients, respectively. No significant difference was noted between the 10 -year actuarial local recurrence rate for the local excision group versus the TME group (10.6\% and $7.6 \%$, respectively; $\mathrm{P}=0.52$ ). The DFS, disease-specific survival, or OS rates also did not differ between the two groups. This study indicated that, in selected patients with cT3 rectal cancer who demonstrated an excellent response to preoperative CRT, full-thickness local excision offered outcomes comparable to those achieved with TME.

However, before the option of surgical conservation can be offered safely to patients receiving preoperative CRT, a randomized study designed specifically to assess the non-inferiority of the conservative approach using local recurrence and OS as the primary endpoints, as well as innovative methods for accurately evaluating post-CRT remnant disease before surgery, are required. Meanwhile, local excision after preoperative CRT is likely to remain a second-choice therapeutic option for LARC patients who are elderly and frail and who are unfit to undergo conventional radical surgery.

\section{NEW RT METHODS}

The preoperative RT scheme (long-course) for LARC usually uses three-dimensional conformal techniques delivering 45 Gy to the tumor and pelvic lymphatics, followed by additional irradiation to the gross tumor to a total dose of $50.4 \mathrm{~Gy}$ in 28 fractions over 5.5 weeks. Concurrently, 5-fluorouracil-based chemotherapy is administered, followed by surgery at 4 to 8 weeks after the completion of CRT. Although various drugs (capecitabine, irinotecan, and oxaliplatin) and molecular targeted therapies (epidermal growth factor receptor inhibitors, vascular endothelial growth factor inhibitors) are being explored as single agents or in combination [46-48], the RT scheme has long been uniformly standardized. Nonetheless, some studies concerning innovative RT methods have been done to enhance the efficacy or to decrease the toxicity. Kim et al. [49] treated 38 LARC patients preoperatively with pelvic RT of 43.2 Gy/24 fractions, and a boost treatment with 7.2 Gy/12 fractions was administered concomitantly during the latter half of pelvic RT. This modified RT fractionation was designed to shorten the treatment period and, thus, escalate the effective dose. However, this concomitant boost irradiation did not improve the clinical outcome compared with conventional preoperative regimens. In other studies, the intensity-modulated RT technique was investigated in preoperative CRT for LARC patients and demonstrated effective reductions in the irradiated small bowel volume and the bowel toxicity [50, 51]. Compared with conventional RT techniques, intensity-modulated RT shows better coverage of the planning target volume and better sparing of organs at risk, and has been rapidly implemented for various tumor types [52]. Much remains to be explored in preoperative RT strategy for rectal cancer regarding dose fractionation (short-course vs. long-course), treatment planning and delivery techniques, interaction with drugs, and molecular modulators of radiation resistance.

\section{CONCLUSION}

Preoperative RT has played a pivotal role in multimodality management for LARC patients. It provides significant benefit in local disease control even with optimal surgical techniques.

Additionally, it permits early recognition of specific tumor biology for each patient. Based on pathologic CRT response identified through surgical pathology, prognosis can be predicted; thus, subsequent postoperative treatment may be tailored. Furthermore, when this pathologic CRT response can be accurately determined before surgery, or even before initiation of CRT, risk-adjusted treatments, such as CRT intensification or conservative surgery, can be recommended for selected patients (Fig. 1). Research on riskadapted strategies for treating LARC patients should be continued to improve tumor control and simultaneously to lower treatment toxicity. 


\section{CONFLICT OF INTEREST}

No potential conflict of interest relevant to this article was reported.

\section{ACKNOWLEDGMENTS}

This work was supported by a National Cancer Center Grant (NCC1210490).

\section{REFERENCES}

1. Kane JM 3rd, Petrelli NJ. Controversies in the surgical management of rectal cancer. Semin Radiat Oncol 2003;13:403-18.

2. Oh JH, Kim DY. Role of radiation therapy as an adjuvant treatment in rectal cancer management. J Korean Soc Coloproctol 2009;25:273-82.

3. Krook JE, Moertel CG, Gunderson LL, Wieand HS, Collins RT, Beart RW, et al. Effective surgical adjuvant therapy for high-risk rectal carcinoma. N Engl J Med 1991;324:709-15.

4. Sauer R, Becker H, Hohenberger W, Rodel C, Wittekind C, Fietkau $\mathrm{R}$, et al. Preoperative versus postoperative chemoradiotherapy for rectal cancer. N Engl J Med 2004;351:1731-40.

5. Colorectal Cancer Collaborative Group. Adjuvant radiotherapy for rectal cancer: a systematic overview of 8,507 patients from 22 randomised trials. Lancet 2001;358:1291-304.

6. Gunderson LL, Haddock MG, Schild SE. Rectal cancer: preoperative versus postoperative irradiation as a component of adjuvant treatment. Semin Radiat Oncol 2003;13:419-32.

7. Roh MS, Colangelo LH, O'Connell MJ, Yothers G, Deutsch M, Allegra CJ, et al. Preoperative multimodality therapy improves disease-free survival in patients with carcinoma of the rectum: NSABP R-03. J Clin Oncol 2009;27:5124-30.

8. Yeo SG, Kim DY, Park JW, Choi HS, Oh JH, Kim SY, et al. Stageto-stage comparison of preoperative and postoperative chemoradiotherapy for T3 mid or distal rectal cancer. Int J Radiat Oncol Biol Phys 2012;82:856-62.

9. Quirke P, Durdey P, Dixon MF, Williams NS. Local recurrence of rectal adenocarcinoma due to inadequate surgical resection. Histopathological study of lateral tumour spread and surgical excision. Lancet 1986;2:996-9.

10. Heald RJ, Moran BJ, Ryall RD, Sexton R, MacFarlane JK. Rectal cancer: the Basingstoke experience of total mesorectal excision, 1978-1997. Arch Surg. 1998;133:894-9.

11. van Gijn W, Marijnen CA, Nagtegaal ID, Kranenbarg EM, Putter $\mathrm{H}$, Wiggers T, et al. Preoperative radiotherapy combined with total mesorectal excision for resectable rectal cancer: 12-year followup of the multicentre, randomised controlled TME trial. Lancet Oncol 2011;12:575-82.

12. Sebag-Montefiore D, Stephens RJ, Steele R, Monson J, Grieve R, Khanna $S$, et al. Preoperative radiotherapy versus selective postoperative chemoradiotherapy in patients with rectal cancer (MRC CR07 and NCIC-CTG C016): a multicentre, randomised trial.
Lancet 2009;373:811-20.

13. Gerard JP, Conroy T, Bonnetain F, Bouche O, Chapet O, ClosonDejardin MT, et al. Preoperative radiotherapy with or without concurrent fluorouracil and leucovorin in T3-4 rectal cancers: results of FFCD 9203. J Clin Oncol 2006;24:4620-5.

14. Bosset JF, Collette L, Calais G, Mineur L, Maingon P, RadosevicJelic L, et al. Chemotherapy with preoperative radiotherapy in rectal cancer. N Engl J Med 2006;355:1114-23.

15. Methy N, Bedenne L, Conroy T, Bouche O, Chapet O, Ducreux M, et al. Surrogate end points for overall survival and local control in neoadjuvant rectal cancer trials: statistical evaluation based on the FFCD 9203 trial. Ann Oncol 2010;21:518-24.

16. Capirci C, Valentini V, Cionini L, De Paoli A, Rodel C, GlynneJones $\mathrm{R}$, et al. Prognostic value of pathologic complete response after neoadjuvant therapy in locally advanced rectal cancer: longterm analysis of 566 ypCR patients. Int J Radiat Oncol Biol Phys 2008;72:99-107.

17. Yeo SG, Kim DY, Kim TH, Chang HJ, Oh JH, Park W, et al. Pathologic complete response of primary tumor following preoperative chemoradiotherapy for locally advanced rectal cancer: long-term outcomes and prognostic significance of pathologic nodal status (KROG 09-01). Ann Surg 2010;252:998-1004.

18. Maas M, Nelemans PJ, Valentini V, Das P, Rodel C, Kuo LJ, et al. Long-term outcome in patients with a pathological complete response after chemoradiation for rectal cancer: a pooled analysis of individual patient data. Lancet Oncol 2010;11:835-44.

19. Kim TH, Chang HJ, Kim DY, Jung KH, Hong YS, Kim SY, et al. Pathologic nodal classification is the most discriminating prognostic factor for disease-free survival in rectal cancer patients treated with preoperative chemoradiotherapy and curative resection. Int J Radiat Oncol Biol Phys 2010;77:1158-65.

20. Kim NK, Baik SH, Seong JS, Kim H, Roh JK, Lee KY, et al. Oncologic outcomes after neoadjuvant chemoradiation followed by curative resection with tumor-specific mesorectal excision for fixed locally advanced rectal cancer: Impact of postirradiated pathologic downstaging on local recurrence and survival. Ann Surg 2006;244:1024-30.

21. Yeo SG, Kim DY. Reply to Letter: 'What does residual nodal positivity in patients with rectal cancer downstaged to ypT0 after chemoradiotherapy really mean?' Ann Surg 2012;255:e2.

22. Moon SH, Kim DY, Park JW, Oh JH, Chang HJ, Kim SY, et al. Can the new American Joint Committee on Cancer staging system predict survival in rectal cancer patients treated with curative surgery following preoperative chemoradiotherapy? Cancer 2012 Mar 13 [Epub]. http://dx.doi.org/10.1002/cncr.27507.

23. Ordoñez C, Screaton RA, Ilantzis C, Stanners CP. Human carcinoembryonic antigen functions as a general inhibitor of anoikis. Cancer Res 2000;60:3419-24.

24. Yoon SM, Kim DY, Kim TH, Jung KH, Chang HJ, Koom WS, et al. Clinical parameters predicting pathologic tumor response after preoperative chemoradiotherapy for rectal cancer. Int J Radiat Oncol Biol Phys 2007;69:1167-72. 
25. Park JW, Lim SB, Kim DY, Jung KH, Hong YS, Chang HJ, et al. Carcinoembryonic antigen as a predictor of pathologic response and a prognostic factor in locally advanced rectal cancer patients treated with preoperative chemoradiotherapy and surgery. Int J Radiat Oncol Biol Phys 2009;74:810-7.

26. Lee SD, Park JW, Park KS, Lim SB, Chang HJ, Kim DY, et al. Influence of anemia on tumor response to preoperative chemoradiotherapy for locally advanced rectal cancer. Int J Colorectal Dis 2009;24:1451-8.

27. Smith FM, Reynolds JV, Miller N, Stephens RB, Kennedy MJ. Pathological and molecular predictors of the response of rectal cancer to neoadjuvant radiochemotherapy. Eur J Surg Oncol 2006; 32:55-64.

28. Chang HJ, Jung KH, Kim DY, Jeong SY, Choi HS, Kim YH, et al. $\mathrm{Bax}$, a predictive marker for therapeutic response to preoperative chemoradiotherapy in patients with rectal carcinoma. Hum Pathol 2005;36:364-71.

29. Min BS, Choi YJ, Pyo HR, Kim H, Seong J, Chung HC, et al. Cyclooxygenase-2 expression in pretreatment biopsy as a predictor of tumor responses after preoperative chemoradiation in rectal cancer. Arch Surg 2008;143:1091-7.

30. Kim IJ, Lim SB, Kang HC, Chang HJ, Ahn SA, Park HW, et al. Microarray gene expression profiling for predicting complete response to preoperative chemoradiotherapy in patients with advanced rectal cancer. Dis Colon Rectum 2007;50:1342-53.

31. Eisenhauer EA, Therasse P, Bogaerts J, Schwartz LH, Sargent D, Ford R, et al. New response evaluation criteria in solid tumours: revised RECIST guideline (version 1.1). Eur J Cancer 2009;45: 228-47.

32. Yeo SG, Kim DY, Kim TH, Jung KH, Hong YS, Chang HJ, et al. Tumor volume reduction rate measured by magnetic resonance volumetry correlated with pathologic tumor response of preoperative chemoradiotherapy for rectal cancer. Int J Radiat Oncol Biol Phys 2010;78:164-71.

33. Yeo SG, Kim DY, Park JW, Oh JH, Kim SY, Chang HJ, et al. Tumor volume reduction rate after preoperative chemoradiotherapy as a prognostic factor in locally advanced rectal cancer. Int J Radiat Oncol Biol Phys 2012;82:e193-9.

34. Kang JH, Kim YC, Kim H, Kim YW, Hur H, Kim JS, et al. Tumor volume changes assessed by three-dimensional magnetic resonance volumetry in rectal cancer patients after preoperative chemoradiation: the impact of the volume reduction ratio on the prediction of pathologic complete response. Int J Radiat Oncol Biol Phys 2010;76:1018-25.

35. Zeestraten EC, Kuppen PJ, van de Velde CJ, Marijnen CA. Prediction in rectal cancer. Semin Radiat Oncol 2012;22:175-83.

36. Hur H, Kim NK, Yun M, Min BS, Lee KY, Keum KC, et al. 18Fluoro-deoxy-glucose positron emission tomography in assessing tumor response to preoperative chemoradiation therapy for locally advanced rectal cancer. J Surg Oncol 2011;103:17-24.

37. Kim YC, Lim JS, Keum KC, Kim KA, Myoung S, Shin SJ, et al. Comparison of diffusion-weighted MRI and MR volumetry in the evaluation of early treatment outcomes after preoperative chemoradiotherapy for locally advanced rectal cancer. J Magn Reson Imaging 2011;34:570-6.

38. Lambrecht M, Deroose C, Roels S, Vandecaveye V, Penninckx F, Sagaert X, et al. The use of FDG-PET/CT and diffusion-weighted magnetic resonance imaging for response prediction before, during and after preoperative chemoradiotherapy for rectal cancer. Acta Oncol 2010;49:956-63.

39. Bentrem DJ, Okabe S, Wong WD, Guillem JG, Weiser MR, Temple LK, et al. T1 adenocarcinoma of the rectum: transanal excision or radical surgery? Ann Surg 2005;242:472-7.

40. Bujko K, Sopylo R, Kepka L. Local excision after radio(chemo) therapy for rectal cancer: is it safe? Clin Oncol (R Coll Radiol) 2007;19:693-700.

41. Read TE, Andujar JE, Caushaj PF, Johnston DR, Dietz DW, Myerson RJ, et al. Neoadjuvant therapy for rectal cancer: histologic response of the primary tumor predicts nodal status. Dis Colon Rectum 2004;47:825-31.

42. Kim DW, Kim DY, Kim TH, Jung KH, Chang HJ, Sohn DK, et al. Is $\mathrm{T}$ classification still correlated with lymph node status after preoperative chemoradiotherapy for rectal cancer? Cancer 2006; 106:1694-700.

43. Lezoche G, Baldarelli M, Guerrieri M, Paganini AM, De Sanctis A, Bartolacci S, et al. A prospective randomized study with a 5-year minimum follow-up evaluation of transanal endoscopic microsurgery versus laparoscopic total mesorectal excision after neoadjuvant therapy. Surg Endosc 2008;22:352-8.

44. Yeo SG, Kim DY, Kim TH, Kim SY, Chang HJ, Park JW, et al. Local excision following pre-operative chemoradiotherapy-induced downstaging for selected cT3 distal rectal cancer. Jpn J Clin Oncol 2010;40:754-60.

45. Callender GG, Das P, Rodriguez-Bigas MA, Skibber JM, Crane $\mathrm{CH}$, Krishnan S, et al. Local excision after preoperative chemoradiation results in an equivalent outcome to total mesorectal excision in selected patients with T3 rectal cancer. Ann Surg Oncol 2010;17:441-7.

46. Kim SY, Hong YS, Kim DY, Kim TW, Kim JH, Im SA, et al. Preoperative chemoradiation with cetuximab, irinotecan, and capecitabine in patients with locally advanced resectable rectal cancer: a multicenter Phase II study. Int J Radiat Oncol Biol Phys 2011;81: 677-83.

47. Kim DY, Jung KH, Kim TH, Kim DW, Chang HJ, Jeong JY, et al. Comparison of 5-fluorouracil/leucovorin and capecitabine in preoperative chemoradiotherapy for locally advanced rectal cancer. Int J Radiat Oncol Biol Phys 2007;67:378-84.

48. Hong YS, Kim DY, Lim SB, Choi HS, Jeong SY, Jeong JY, et al. Preoperative chemoradiation with irinotecan and capecitabine in patients with locally advanced resectable rectal cancer: long-term results of a Phase II study. Int J Radiat Oncol Biol Phys 2011;79: 1171-8.

49. Kim DY, Kim TH, Jung KH, Chang HJ, Lim SB, Choi HS, et al. Preoperative chemoradiotherapy with concomitant small field 
boost irradiation for locally advanced rectal cancer: a multi-institutional phase II study (KROG 04-01). Dis Colon Rectum 2006; 49:1684-91.

50. Kim JY, Kim DY, Kim TH, Park SY, Lee SB, Shin KH, et al. Intensity-modulated radiotherapy with a belly board for rectal cancer. Int J Colorectal Dis 2007;22:373-9.

51. Samuelian JM, Callister MD, Ashman JB, Young-Fadok TM, Borad
MJ, Gunderson LL. Reduced acute bowel toxicity in patients treated with intensity-modulated radiotherapy for rectal cancer. Int J Radiat Oncol Biol Phys 2012;82:1981-7.

52. Veldeman L, Madani I, Hulstaert F, De Meerleer G, Mareel M, De Neve W. Evidence behind use of intensity-modulated radiotherapy: a systematic review of comparative clinical studies. Lancet Oncol 2008;9:367-75. 